Phase transf or mat i on of mesopor ous cal ci um carbonate by mechanical stirring

\begin{tabular}{|l|l|}
\hline 著者 & $\begin{array}{l}\text { YAMANAKA Shi nya, SUGAWARA Yut o, O SO Takahi ro, } \\
\text { FUJI MOTO Toshi yuki, OHI RA Yui chi, KUGA } \\
\text { Yoshi kazu }\end{array}$ \\
\hline $\begin{array}{l}\text { j our nal or } \\
\text { publ i cat i on ti t e }\end{array}$ & Cryst EngComm \\
\hline vol une & 17 \\
\hline number & 8 \\
\hline page range & $1773-1777$ \\
\hline year & $2015-01-21$ \\
\hline URL & ht t p: //hdl . handl e. net /10258/00009470 \\
\hline
\end{tabular}




\title{
Phase transformation of mesoporous calcium carbonate by mechanical stirring
}

\author{
Shinya Yamanaka, ${ }^{* a}$ Yuto Sugawara, ${ }^{b}$ Takahiro Oiso, ${ }^{b}$ Toshiyuki Fujimoto, ${ }^{a}$ Yuichi Ohira ${ }^{a}$ and \\ Yoshikazu Kuga ${ }^{a}$
}

Received (in $X X X, X X X)$ Xth $X X X X X X X X X 20 X X$, Accepted Xth $X X X X X X X X X 20 X X$

DOI: $10.1039 / b 000000 x$

We report a simple strategy to synthesize vaterite/calcite mesoporous calcium carbonate through collisions and 10 organization of colloid particles accelerated by mechanical stirring. Mechanically stirring the precursor colloidal dispersion can control the calcium carbonate polymorphs.

Due to the diverse applications of calcium carbonate (e.g., controllable release and encapsulation of drugs, ${ }^{1-3}$ catalyst 15 support, ${ }^{4}$ gas adsorbent, ${ }^{5}$ and abiotic and biogenic mineral formation $^{6-8}$ ), polymorphic selectivity or pore formation of calcium carbonate is of considerable interest. However, controlling the polymorphs during large-scale productions of porous calcium carbonate remains challenging. Crystalline 20 calcium carbonate polymorphs of calcite, aragonite, and vaterite are thought to crystallize via unstable amorphous calcium carbonate (ACC). ${ }^{6,7,9,10}$

Classical nucleation theory, which is based on the works of Gibbs, ${ }^{11}$ Volmer and Weber, ${ }^{12,}{ }^{13}$ Becker and Doring, ${ }^{14}$ and

${ }_{25}$ Frenkel, ${ }^{15}$ stipulates that the birth of a crystal in solution is governed by the free energy barrier, which corresponds to the energy required to generate a critical nucleus. Visible and stable crystals undergo a pre-nucleation stage ${ }^{16}$ due to cluster fluctuations, critical nuclei generation, and subsequent growth of 30 nuclei. Clusters, which are widely thought to be the origin of nucleation or crystal birth, ${ }^{16}$ have been observed experimentally. ${ }^{13,17-19}$

These pre-nucleation clusters are often observed during precipitation of crystalline calcium carbonate. Initially formed $35 \mathrm{ACC}$ is a transient phase, which is subsequently transformed into more stable crystalline polymorphs according to Ostwald's rule. ${ }^{16}$ Because the transformation of ACC into crystalline calcium carbonate depends on the thermodynamic equilibrium, the thermal history ${ }^{20,} 21$ and the water content ${ }^{22}$ have been 40 investigated to determine the kinetics and mechanism of the ACC transformation. Rodriguez-Blanco et al. have proposed a twostage crystallization process in an aqueous solution ${ }^{23}$; in the first stage, rapid dehydration of ACC forms the vaterite phase, whereas dissolution of vaterite and subsequent reprecipitation 45 forms calcite. That is, by tuning the ACC stability, crystallization of calcium carbonate can be controlled. ${ }^{10,24,25}$ For example, to obtain stable vaterite, several additives (e.g., ethanol, ${ }^{26}$ dopamine, ${ }^{27}$ dendrimers, ${ }^{28,}{ }^{29}$ poly acrylic acid,${ }^{30}$ and mesoporous silica $^{31}$ ) have been studied to prevent the phase transformation 50 into calcite. In addition to polymorphic considerations, the formation of pores in calcium carbonate crystals has advanced. Porous calcium carbonate is produced mainly via heterogeneous nucleation on templates. ${ }^{32-35}$

Here we report a scalable production strategy for mesoporous 55 calcium carbonate that controls the polymorphs. Mechanical stirring accelerates the incorporation of pores via collisions of colloid particles. Applications of mechanical stirring to the colloidal dispersion confirm that an energy-induced phase transformation can convert ACC to vaterite or calcite.

${ }_{60} \mathrm{We}$ prepared a transparent colloidal dispersion, which is a precursor for porous calcium carbonate (see ESI†े). Slight mechanical stirring during the pre-aging process affects the aggregation route of the colloid particles. Without agitation, the transparent supernatant requires one-month to become a cloudy ${ }_{65} \mathrm{sol}$, but mixing reduces the transformation time. For example, the cloudy sol is obtained two days after stirring the dispersion for 24 hours. These observations suggest that agitation accelerates nanoparticle collisions, ${ }^{36}$ resulting in organization of porous calcium carbonate particles in a shorter amount of time.

70 We initially characterized the transparent colloidal dispersion. Dynamic light scattering (DLS) analysis was conducted to determine the size distribution of the colloidal particles. The DLS

80

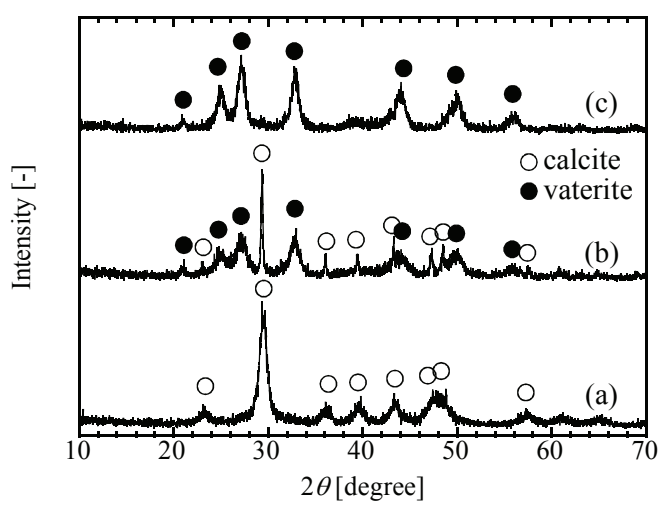

Figure 1. Typical XRD patterns of the prepared particles after an aging treatment. (a) Sample obtained without stirring. Crystallite size of $13 \mathrm{~nm}$ for (104) calcite roughly coincides with the primary particle size from the DLS median size of $19.5 \mathrm{~nm}$. Samples 90 obtained after stirring for (b) 12 hours and (c) at 24 hours. 

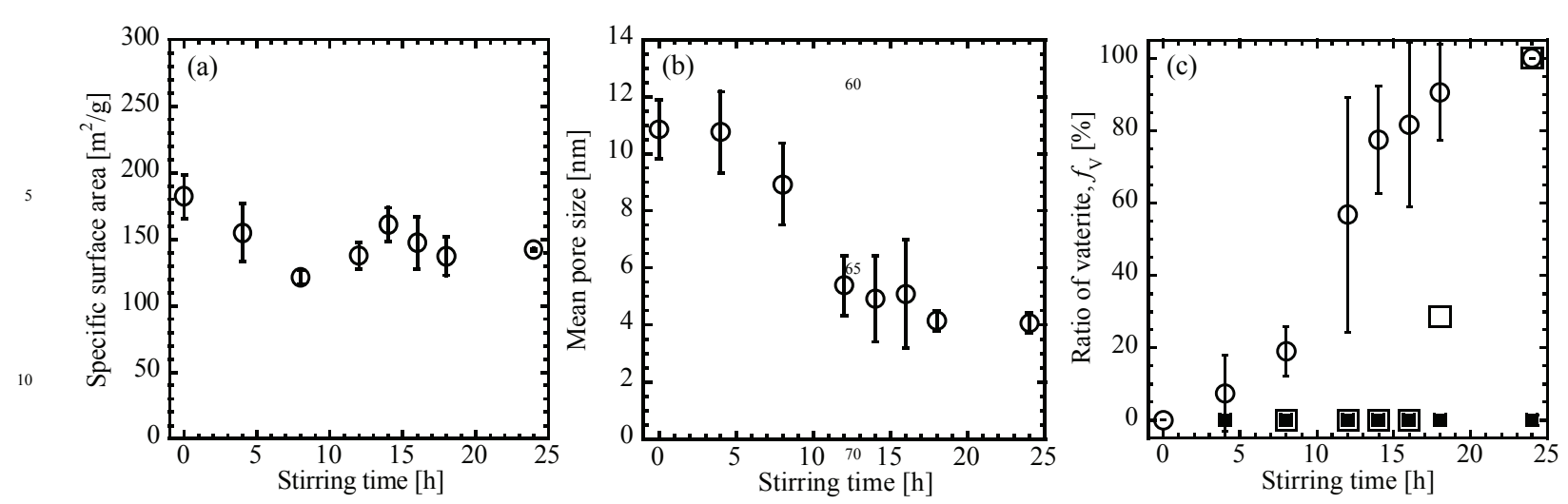

Figure 2. Select features of the samples obtained by mechanical energy induction. Mechanical stirring was continuous for 0 to 24 hours. (a) Specific surface area, (b) BJH mean pore size measured from the nitrogen isotherm plots, and (c) effect of the stirring rate, stirring time, and stirring method on the porous calcium carbonate polymorphs. Samples obtained after stirring at $300 \mathrm{rpm}$ (open circles), $10 \mathrm{rpm}$ (open squares), and shaking by a reciprocál shaker at $100 \mathrm{rpm}$ (closed squares). Ratio of vaterite crystal was calculated from Rao's equation. ${ }^{37}$

20 median size for the colloid particles is $19.5 \mathrm{~nm}$ (see ESI $\dagger$, Fig. S1). The solid fraction calculated from the weight loss of the dispersion using TG-DTA is $0.57 \pm 0.03$ mass\% (indicating a stoichiometric yield of $84 \pm 5 \%$ ). Mechanical stirring for a predetermined time results in rapid aggregation of the colloid ${ }_{25}$ particles.

Next we investigated the particle properties of porous calcium carbonate. The peak positions of the XRD profiles correspond to those of the calcite (ICDD\#05-0586) and/or vaterite (ICDD\#330268) phase for all samples (Fig. 1). Diffraction indicates that the 30 calcite phase is formed without stirring the colloidal dispersion (Fig. 1a). When pre-aging is carried out, the amount of the vaterite increases with stirring time (Fig. 1b). Only the vaterite phase is detected after stirring for 24 hours, indicating that the calcite phase completely disappears (Fig. 1c).

${ }_{35}$ Regardless of the phase, all the profile peaks are relatively weak and broad, suggesting a small crystallite size. The crystallite size of porous calcium carbonate was calculated from the full width at half maximum (FWHM) of the corrected diffraction profile. A Pseudo-Voigt fitting was conducted to obtain the FWHM for the 40 (104) calcite diffraction. If the precursor dispersion is not agitated during the pre-aging treatment, the crystallite size is $13 \mathrm{~nm}$ for (104) calcite, which is close to the colloid particle size of the DLS median $(19.5 \mathrm{~nm})$, indicating that pores may form between the aggregated primary particles.

45 Figure 2 shows the specific surface area, average pore size, and ratio of vaterite to calcite crystal $\left(f_{\mathrm{V}}\right)$ as functions of stirring time. $f_{\mathrm{V}}$ was calculated using Rao's equation ${ }^{37}$ as

50

$$
f_{\mathrm{V}}=\frac{I_{110 \mathrm{~V}}+I_{112 \mathrm{~V}}+I_{114 \mathrm{~V}}}{I_{110 \mathrm{~V}}+I_{112 \mathrm{~V}}+I_{114 \mathrm{~V}}+I_{104 \mathrm{C}}}
$$

where the subscripts $\mathrm{V}$ and $\mathrm{C}$ indicate the intensity for each vaterite and calcite crystal face, respectively. The specific surface area of porous calcium carbonate is $148 \pm 22 \mathrm{~m}^{2} / \mathrm{g}$ (Fig. 2a), regardless of stirring time. The average pore size and $f_{\mathrm{V}}$ depend 55 on the stirring time (Figs. 2b, c). For short stirring times (less than 12 hours), calcite is the main crystal phase. As the stirring time increases up to 12 hours, $f_{\mathrm{V}}$ drastically increases and the average pore size decreases from $\sim 10 \mathrm{~nm}$ to $\sim 4 \mathrm{~nm}$.
We have previously reported a self-assembly route for porous calcium carbonate, which is associated with the phase 80 transformation from ACC to calcite and/or vaterite, and demonstrated the effect of aging temperature on the morphology of porous calcium carbonate (i.e., thermal energy leads to Brownian aggregation of the colloid particles). ${ }^{38}$ Consequently, the colloid aggregates form more preferred structures due to the ${ }_{85}$ reduced surface free energy. ${ }^{39}$

The solubility of calcium carbonate is crucial for the transformation. $^{38,} 40$ The FTIR spectra (see ESI†, Fig. S2) and XRD (see ESI†, Fig. S3) suggest that ACCs are dispersed in an organic solvent mixture as well as contained in mesoporous 90 calcium carbonate particles. Although the carbonation process in this study contains little water, it stoichiometrically produces equimolar amounts of water and calcium carbonate. To obtain the calcite phase of mesoporous calcium carbonate, a long aging process (one month) is required. During the aging treatment, 95 dissolution of the vaterite and subsequent reprecipitation forms calcite. The FTIR studies reveal that ethylene glycol remains on the surface (see ESI†, Fig. S2), which may prevent vaterite dissolution and calcite transformation. Thus, the water content in the transparent supernatant (colloidal dispersion) should play an 100 important role in the polymorphs.

We also added water (1.0-10.0 mass\%) to the colloidal dispersion $(9: 1-100: 1$ by the molar ratio of water and calcium carbonate). After 24 hours of agitation and a subsequent aging treatment, the ratio of vaterite phase was determined. As shown 105 in Fig. 3, adding over 3.0 mass $\%$ water decreases the ratio of vaterite, indicating that the vaterite crystal dissolves in water to form the calcite phase.

Previous studies have investigated the effect of the stirring rate on controlling the crystal morphology of calcium carbonate; they 110 have demonstrated that adjusting the stirring rate tunes the vaterite and/or calcite phase. ${ }^{41,42}$ To investigate the true role of stirring in the system, we examined the influences of stirring rate and stirring method on the polymorphs using two different stirring rates (10 and $300 \mathrm{rpm})$ and a reciprocal shaker $(100 \mathrm{rpm})$, 115 respectively. Figure $2 \mathrm{c}$ plots the results. Although the appearance time of vaterite phase $\left(f_{\mathrm{V}}>0\right)$ for 10 -rpm stirring is delayed 


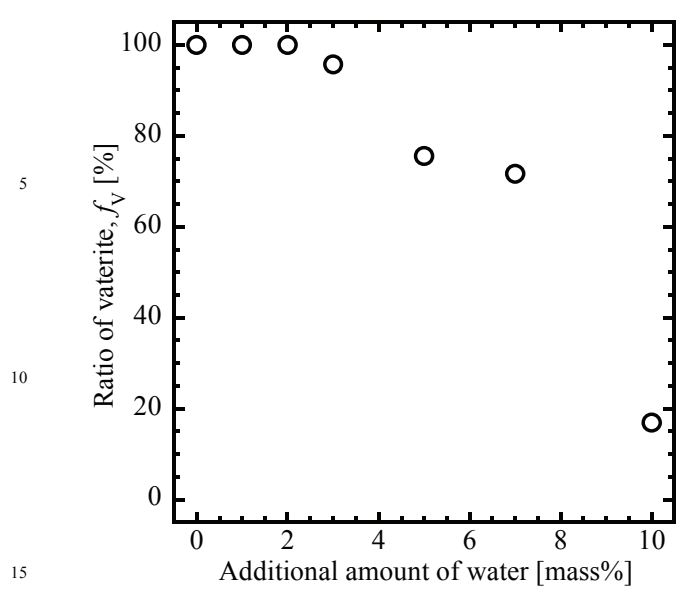

Figure 3. Effect of water addition on the ratio of the vaterite phase. Water (1.0-10.0 mass\%) is added to the colloidal dispersion (corresponding to 9:1-100:1 by molar ratio of water and calcium carbonate) before agitation for 24-hours and a 20 subsequent aging treatment.

compared to 300 -rpm stirring, only the vaterite phase $\left(f_{\mathrm{V}}=1.0\right)$ is obtained after stirring for 24 hours. The agitation Reynolds number $(R e)$ for $10(300) \mathrm{rpm}$ stirring rate is 140 (4000) $25\left(R e=\rho \cdot n \cdot d^{2} / \mu\right.$, where $\rho=900 \mathrm{~kg} / \mathrm{m}^{3}$ is the density of dispersion, $n=1.7 \times 10^{-1}(5.0) \mathrm{s}^{-1}$ for a stirring rate of $10(300) \mathrm{rpm}, d=5.0 \times 10^{-2}$ $\mathrm{m}$ is the impeller diameter, and $\mu=2.78 \times 10^{-3} \mathrm{~Pa} \cdot \mathrm{s}$ is the viscosity of the colloidal dispersion). Although the pre-aging treatment was conducted in the laminar and turbulent flow, this range of stirring

30 has a negligible effect on the kinetics of vaterite formation. The required time to obtain the vaterite phase is the same, indicating that the transformation of ACC to the vaterite phase is accomplished by adding an imperceptible but continuous energy fluctuation from the agitation impeller to the precursor colloids. 35 Although more than 12 hours of stirring is necessary to produce the vaterite phase, the crystalline phase transformation requires less time (minutes or hours). ${ }^{23,31}$ Shaking the colloidal dispersion using a reciprocal shaker at $100 \mathrm{rpm}$ yields only the calcite phase after 24 hours (see Fig. 2(c) and ESI†, Fig. S4).

${ }_{40}$ In the future, we should examine the density of water molecules around the colloids because the water addition tests suggest that dissolution and reprecipitation may occur during the pre-aging treatment. The effects of the flow condition and water content on the polymorphic transformation need to be further investigated.

${ }_{45}$ As mentioned above, the FTIR spectra demonstrate mesoporous calcium carbonate particles include ACCs (see ESI†, Fig. S2). ACC typically has a high specific surface area with $140-160 \mathrm{~m}^{2} / \mathrm{g}$ ${ }^{43}$ and $100-300 \mathrm{~m}^{2} / \mathrm{g}{ }^{44}$. Thus, the high specific surface area $\left(148 \pm 22 \mathrm{~m}^{2} / \mathrm{g}\right)$ of the mesoporous calcium carbonate is due to the ${ }_{50} \mathrm{ACC}$ in its structure. Although the actual particle size from the SEM images in Fig. 4 differs from its crystallite size (13 nm for calcite (104) and $10 \mathrm{~nm}$ for vaterite (112)), the calculated equivalent diameter is $14-24 \mathrm{~nm}$, which is consistent with the crystallite size. The equivalent spherical diameter is equal to ${ }_{55} 6 /\left(\rho_{\mathrm{p}} \times \mathrm{SSA}\right)$, where $\rho_{\mathrm{p}}=2000-2500 \mathrm{~kg} / \mathrm{m}^{3}$ is the density of ACC ${ }^{44}$ and $\mathrm{SSA}=148 \pm 22 \mathrm{~m}^{2} / \mathrm{g}$ is the specific surface area from the BET measurement. The pores in mesoporous calcium carbonate are formed by void spaces between the primary particles.
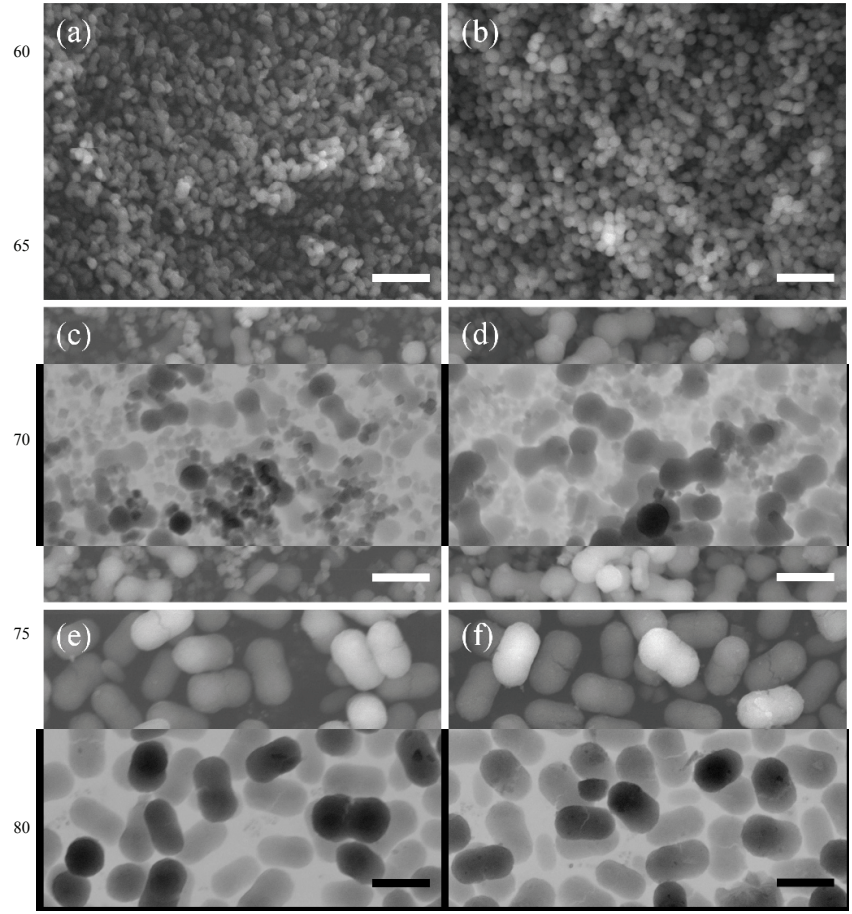

Figure 4. Typical SEM images of the porous calcium carbonate particles obtained with stirring for (a) 0 hours, (b) 4 hours, (c) 8 85 hours, (d) 12 hours, (e) 18 hours, and (f) 24 hours. Scale bar is 2 $\mu \mathrm{m}$. The stirring rate was $300 \mathrm{rpm}$.

Figure 4 depicts typical SEM images of the porous calcium carbonate particles for different stirring times $(0,4,8,12,18$, and 9024 hours) at a stirring rate of $300 \mathrm{rpm}$. The porous particles assume one of two shapes: tiny $(\sim 300 \mathrm{~nm})$ almost-spherical particles or relatively large ellipsoidal one. The major and minor axis sizes for the ellipsoidal shape of vaterite calcium carbonate are 2 and $1 \mu \mathrm{m}$, respectively. Spherical particles form without or ${ }_{95}$ with a mechanical stirring for a short time (Figs. $4 \mathrm{a}, \mathrm{b}$ ), but once a certain moment is reached, an ellipsoidal structure is obtained (Figs. 4e, f). This transition occurs around 8 and 12 hours of stirring, and both shapes are observed (Figs. 4c, d). Stirring for 12 hours coincides with the anticipate variations in the pore and 100 crystallographic structure from Fig. 2.

To investigate the effect of mechanical stirring on the transition period, we conducted an experiment where the sample was stirred at $10 \mathrm{rpm}$ or shaken at $100 \mathrm{rpm}$. The time required for the only vaterite phase to appear is at about the same ( 24 hours stirring) 105 regardless of the stirring rate (10 or $300 \mathrm{rpm})$. However, shaking did not produce the vaterite phase, indicating that the flow condition is crucial in the transformation of metastable vaterite to stable calcite. These findings suggest that porous calcium carbonate may be a promising adsorbent material because the 110 vaterite structure can easily adsorb formaldehyde. ${ }^{38}$

\section{Conclusions}

This study demonstrates the production method for mesoporous calcium carbonate for two individual polymorphs: vaterite and 115 calcite. The influence of mechanical stirring on the polymorphic 
transformation was investigated. Applying continuous agitation into the colloidal intermediate dispersion forms pores in the crystal via organization of the colloid particles. The mechanical stirring time influences not only the polymorphs, but also the rate 5 of organization. The water content in the colloidal dispersion plays a crucial role in the transformation from vaterite to calcite.

We thank Prof. Jusuke Hidaka at Doshisha University and Prof. Masanori Yoshida at Muroran Institute of Technology for their 10 technical comments and assistance. This work was supported by a Grant-in-Aid for Scientific Research (C) (No. 25420797) of the Japan Society for the Promotion of Science, and was conducted under the Cooperative Research Program of Institute for Joining and Welding Research Institute, Osaka University.

\section{${ }_{15}$ Notes and references}

${ }^{a}$ College of Environmental Technology, Muroran Institute of Technology, Mizumoto-cho 27-1, Muroran 050-8585, Japan. Fax:+81-143-46-5701;

Tel: +81-143-46-5747; E-mail: syama@mmm.muroran-it.ac.jp

${ }^{b}$ Division of Applied Sciences, Muroran Institute of Technology,

20 Mizumoto-cho 27-1, Muroran 050-8585, Japan

† Electronic Supplementary Information (ESI) available: [Experimental details. Size distributions of colloidal calcium carbonate determined by DLS analysis (Fig. S1). FTIR spectra for colloidal dispersion and mesoporous powder (Fig. S2). XRD pattern for the solid sample separated

25 from the dispersion (Fig. S3), and mesoporous calcium carbonate obtained using a reciprocal shaker (Fig. S4)].

See DOI: $10.1039 / \mathrm{b} 000000 \mathrm{x} /$

1 G. B. Sukhorukov, D.V. Volodkin, A. M. Günther, A. I. Petrov, D. B. Shenoy and H. Mohwald, J. Mater. Chem., 2004, 14, 2073.

2 W. Wei, G. H. Ma, G. Hu, D. Yu, T. Mcleish, Z. G. Su and Z. Y. Shen, J. Am. Chem. Soc., 2008, 130, 15808.

3 M. Fujiwara, K. Shiokawa, M. Araki, N. Ashitaka, K. Morigaki, T. Kubota and Y. Nakahara, Cryst. Growth Des., 2010, 10, 4030.

354 M. García-Mota, J. Gómez-Díaz, G. Novell-Leruth, C. VargasFuentes, L. Bellarosa, B. Bridier, J. Pérez-Ramírez and N. López, Theor. Chem. Acc., 2011, 128, 663.

5 Z. Zhao, L. Zhang, H. Dai, Y. Du, X. Meng, R. Zhang, Y. Liu and J. Deng, Microporous Mesoporous Mater., 2011, 138, 191.

406 L. Addadi, S. Raz and S. Weiner, Adv. Mater., 2003, 15, 959.

7 M. Faatz, F. Grohn and G. Wegner, Adv. Mater., 2004, 16, 996.

8 A. W. Xu, Y. Ma and H. Cölfen, J. Mater. Chem., 2007, 17, 415.

9 H. Cölfen and S. Mann, Angew. Chem. Int. Ed., 2003, 42, 2350.

10 J. Ihli, W. C. Wong, E. H. Noel, Y. Y. Kim, A. N. Kulak, H. K. Christenson, M. J. Duer and F. C. Meldrum, Nat. Commun., 2014 5, 3169 .

11 J. W. Gibbs, Collected works, Vol I, Thermodynamics; Yale University Press: New Haven, USA 1948

12 M. Volmer and Weber A., Z. Pllys. Chem., 1926, 119, 277.

5013 M. Volmer, Z. Elektrochem., 1929, 35, 555.

14 R. Becker and W. Doring, Ann. Phys., 1935, 24, 719.

15 J. Frenkel, Kinetic Theory of Liquids; Dover: New York, USA, 1955

16 D. Gebauer, M. Kellermeier, J. D. Gale, L. Bergströmc and H. Cölfen, Chem. Soc. Rev., 2014, 43, 2348.

5517 R. M. Ginde and A. S. Myerson, J. Cryst. Growth, 1992, 116, 41.

18 J. W. Mullin and C. L. Leci, Phil. Mag., 1969, 19, 1075.

19 A. T. Allen, M. P. McDonald, W. M. Nicol and R. M. Wood, Nature, 1972, 235, 36 .

20 T. Ogino, T. Suzuki and K. Sawada, Geochim. Cosmochim. Acta, 1987, 51, 2757.

21 A. V. Radha, T. Z. Forbes, C. E. Killian, P. U. P. A. Gilbert and A. Navrotsky, Proc. Natl. Acad. Sci. U.S.A., 2010, 107, 16438.

22 M. Ryu, J. Ahn, K. You, S. Goto and H. Kim, J. Ceram. Soc. Japan, 2009, 117, 106.

${ }_{65} 23$ J. D. Rodriguez-Blanco, S. Shaw and L. G. Benning, Nanoscale, $2011,3,265$.
24 J. Ihli, Y. Y. Kim, E. H. Noel and F. C. Meldrum, Adv. Funct. Mater., 2013, 23, 1575

25 T. Y. J. Han and J. Aizenberg, J. Chem. Mater., 2008, 20, 1064.

7026 F. Manoli and E. Dalas, J. Crystal Growth, 2000, 218, 359.

27 S. Kim and C. B. Park, Langmuir, 2010, 26, 14730.

28 K. Naka, Y. Tanaka and Y. Chujo, Langmuir, 2002, 18, 3655.

29 K. Naka, Y. Tanaka, Y. Chujo and Y. Ito, Chem. Commun., 1999, 19, 1931.

7530 K. Naka, S. C. Huang and Y. Chujo, Langmuir, 2006, 22, 7760

31 K. M. Choi and K. Kuroda, Cryst. Growth Des., 2012, 12, 887.

32 D. Walsh and S. Mann, Nature, 1995, 377, 320.

33 T. Tomioka, M. Fuji, M. Takahashi, C. Takai and M. Utsuno, Cryst. Growth Des., 2011, 12, 771.

8034 L. Qi, J. Li and J. Ma, Adv. Mater., 2002, 14, 300.

35 D. Yang, L. Qi and J. Ma, Chem. Commun., 2003, 21, 1180.

36 G. R. Zeichner and W. R. Schowalter, AIChE Journal, 1977, 23, 243.

37 M. S. Rao, Bull. Chem. Soc. Jpn., 1973, 46, 1414.

38 S. Yamanaka, T. Oiso, Y. Kurahashi, H. Abe, K. Hara, T. Fujimoto and Y. Kuga, J. Nanoparticle Res., 2014, 16, 2266.

39 X. H. Guo, Y. H. Deng, B. Tu and D. Y. Zhao, Langmuir, 2010, 26, 702 .

40 G. Hadiko, Y. S. Han, M. Fuji, M. Takahashi, Mater. Lett., 2005, 59, 2519.

9041 R. K. Pai, K. Jansson and N. Hedin, Cryst. Growth Des., 2009, 9, 4581.

42 F. W. Yan, S. F. Zhang, C. Y. Guo, X. H. Zhang, G. C. Chen, F. Yan and G. O. Yuan, Cryst. Res.Technol., 2009, 44, 725.

43 D. Gebauer, X. Liu, B. Aziz, N. Hedin and Z. Zhao, CrystEngComm, 2013, 15, 1257.

44 T. Yasue, A. Mamiya, T. Fukushima and Y. Arai, Gypsum \& Lime, 1985, 198, 245.

4 | Journal Name, [year], [vol], 00-00

This journal is () The Royal Society of Chemistry [year] 


\section{Electronic Supplementary Information}

\section{Phase transformation of mesoporous calcium carbonate by mechanical stirring}

Shinya Yamanaka, ${ }^{* a}$ Yuto Sugawara, ${ }^{b}$ Takahiro Oiso, ${ }^{b}$ Toshiyuki Fujimoto, ${ }^{a}$ Yuichi Ohira ${ }^{a}$ and Yoshikazu Kuga ${ }^{a}$

${ }^{a}$ College of Environmental Technology, Muroran Institute of Technology, Mizumoto-cho 27-1, Muroran 050-8585, Japan

${ }^{b}$ Division of Applied Sciences, Muroran Institute of Technology, Mizumoto-cho 27-1, Muroran 050-8585, Japan

Author to whom correspondence should be addressed (syama@mmm.muroran-it.ac.jp).

\section{Materials}

Calcium hydroxide (Nacalai Tesque Inc., Japan), ethylene glycol (Kanto Chemical, Japan), ethanol (Kanto Chemical, Japan), and calcite calcium carbonate (Nacalai Tesque, Japan) were guaranteed reagents and used as received.

\section{Synthesis of porous calcium carbonate}

A colloidal dispersion of calcium carbonate nanoparticles, which is the liquid precursor for meso-porous calcium carbonate particles, was prepared by carbonation of calcium hydroxide in an ethylene glycol and ethanol (3:7 by weight) organic solvent 
mixture $[1,2]$. The synthetic procedure for the colloidal dispersion is described in detail elsewhere [3]. Briefly, calcium hydroxide (2.5 g) was mixed with an organic mixture (497.5 g) and reacted with 30 vol\% $\mathrm{CO}_{2}$ diluted with $\mathrm{N}_{2}$. During the carbonation reaction, the solution was stirred at a rate of $400 \mathrm{rpm}$ with a gas flow rate of $1.0 \mathrm{l} / \mathrm{min}$. A water bath maintained the temperature at $293 \mathrm{~K}$. After $90 \mathrm{~min}$, the suspension was centrifuged at 3,500 rpm for $20 \mathrm{~min}$ (Model 5800, Kubota, Japan). From the collected supernatant, the transparent dispersion was used as the raw liquid material in the aging treatments. Dynamic light scattering analysis (DLS, Nanotrac UPA, Nikkiso, Japan) was conducted to determine the colloidal particle size distribution (Fig. S1). Prior to the DLS analysis, the transparent dispersion was immersed in an ultrasonic bath for $5 \mathrm{~min}$ to ensure homogeneity. The viscosity of the ethylene glycol and ethanol mixture (3:7 by weight), which was used as the parameter in the DLS analysis, was measured using a cone-plate rheometer (RheoStress600, HAAKE, Germany) at 293 and $303 \mathrm{~K}$. The plates had a 50-mm diameter and a fixed gap distance of $52 \mu \mathrm{m}$. The viscosity of the mixture at $293(303) \mathrm{K}$ is $2.60(2.10) \mathrm{mPa} \cdot \mathrm{s}$. To calculate the agitation Reynolds number $(R e)$, the viscosity of the colloidal dispersion was also measured; it is $2.78 \mathrm{mPa} \cdot \mathrm{s}$ at $293 \mathrm{~K}$. The solid fraction of the dispersion was measured from the weight loss at $773 \mathrm{~K}$ using thermogravimetric-differential thermal analysis (TG-DTA, Exstar 6200N, Seiko Instruments, Japan). TG-DTA measurements were conducted with a nitrogen flow of $50 \mathrm{ml} / \mathrm{min}$ at a ramp of $10 \mathrm{~K} / \mathrm{min}$.

To investigate the effect of mechanical stirring on the morphology of mesoporous calcium carbonate, two-step aging treatments were carried out for the resulting transparent supernatant. The first step was pre-aging in which the transparent supernatant was stirred using a propeller-type impeller $(0.05 \mathrm{~m}$ in diameter $)$ or a reciprocal shaker 
(NEO-SHAKER NS-LR, AS ONE). In the former case, the colloidal dispersion was stirred at 10 or $300 \mathrm{rpm}$ for $0-24$ hours ( 0 hours indicates no stirring), while in the latter case, the dispersion was shook at $100 \mathrm{rpm}$ for 0-24 hours. After pre-aging, the colloidal dispersion was placed into an incubator for a predetermined time. Both treatments were conducted at 293 K. The cloudy suspension was washed twice with ethanol to eliminate the excess of ethylene glycol. The suspension was then centrifuged at 3,500 rpm for $15 \mathrm{~min}$. The supernatant was discarded, and the residue was dried in vacuum for 24 hours.

\section{Powder characterization}

The resulting dry powder was used in the measurements. The specific surface area and pore size distribution of the powder were determined by nitrogen gas adsorption based on multi-point BET and the BJH method (Autosorb-1-c/MK2, Qantachrome, USA). In the BET and pore size distribution measurements, the powder was degassed for 2 hours at 473 $\mathrm{K}$ under a vacuum to remove adsorbed solvent molecules. To determine the polymorphs of calcium carbonate, X-ray diffraction (XRD, MultiFlex, Rigaku, Japan) powder patterns of the samples were obtained with $\mathrm{Cu} \mathrm{K} \alpha$ radiation $(40 \mathrm{kV}, 40 \mathrm{~mA})$. SEM studies were performed using a JEOL JSM-6380A.

\section{Initial phase of calcium carbonate}

FTIR spectra were measured by an FT/IR-460PlusK (JASCO) spectrometer with the $\mathrm{KBr}$ pellet technique ranging from 600 to $2000 \mathrm{~cm}^{-1}$. The $\mathrm{KBr}$ pellets contained $1-2$ mass $\%$ of the colloidal dispersion, the resultant precipitates (calcite and vaterite), and reagent calcite powder. Figure S2 shows the IR spectra of the colloidal dispersion, resultant 
precipitates (calcite and vaterite), and regent calcite powder. Amorphous calcium carbonate (ACC) contains water in its structure. ${ }^{4}$ The absorption peak at $1640 \mathrm{~cm}^{-1}(\mathrm{HOH}$ bending) except reagent calcite, are due to ACC. Except for the colloidal dispersion, the internal modes of the carbonate ion in calcite $\left(710 \mathrm{~cm}^{-1}\right)$ or vaterite $\left(740 \mathrm{~cm}^{-1}\right)$ are detected. These data suggest that ACCs are dispersed in the organic solvent mixture and are contained in the mesoporous calcium carbonate particles. The splitting bands that originate from ethylene glycol in the FTIR spectra at $864,882 \mathrm{~cm}^{-1}$ (CC stretching, $\mathrm{CH}_{2}$ rocking), 1040, $1085 \mathrm{~cm}^{-1}$ (CO stretching), and 1410, $1462 \mathrm{~cm}^{-1}$ (COC bending, $\mathrm{CH}_{2}$ bending) reveal that ethylene glycol remains on the surface of mesoporous calcium carbonate. ${ }^{5}$

To identify the initial colloid phase in the dispersion, we conducted an XRD study of the separated solid sample from the dispersion. Because the sample obtained by above process (see Synthesis of porous calcium carbonate) is small in amount, we raised the solid concentration of calcium carbonate in the dispersion. Calcium hydroxide $(25 \mathrm{~g})$ was mixed with an organic mixture (475 g) [3]. The conditions of the carbonation reaction, and the collection method of the colloidal dispersion were the same as that described in Synthesis of porous calcium carbonate. Then, the colloidal dispersion was directly dried in vacuum overnight, washed twice with ethanol, and then centrifuged at 3,500 rpm for 15 min. The supernatant was discarded, and the residue was dried in vacuum overnight. The XRD profile of this separated solid lacks peaks (Fig. S3), indicating the initial phase is ACC. 


\section{References}

[1] Yasue, T.; Mamiya, A.; Fukushima, T.; Arai, Y. Gypsum \& Lime 1985, 198, 245.

[2] Ryu, M.; Ahn, J.; You, K.; Goto, S.; Kim, H.; J. Ceram. Soc. Japan 2009, 117, 106.

[3] Yamanaka, S.; Oiso, T.; Kurahashi, Y.; Abe, H.; Hara, K.; Fujimoto, T.; Kuga, Y. J. Nanoparticle Res. 2014, 16, 2266

[4] Andersen F. A.; Brečević L., Acta. Chem. Scand., 1991, 45, 1018.

[5] Krishnan K.; F. A. Sc. Krishnan R. S., Proc. Indian Acad. Sci. Sect. A, 1966, 64, 111. 


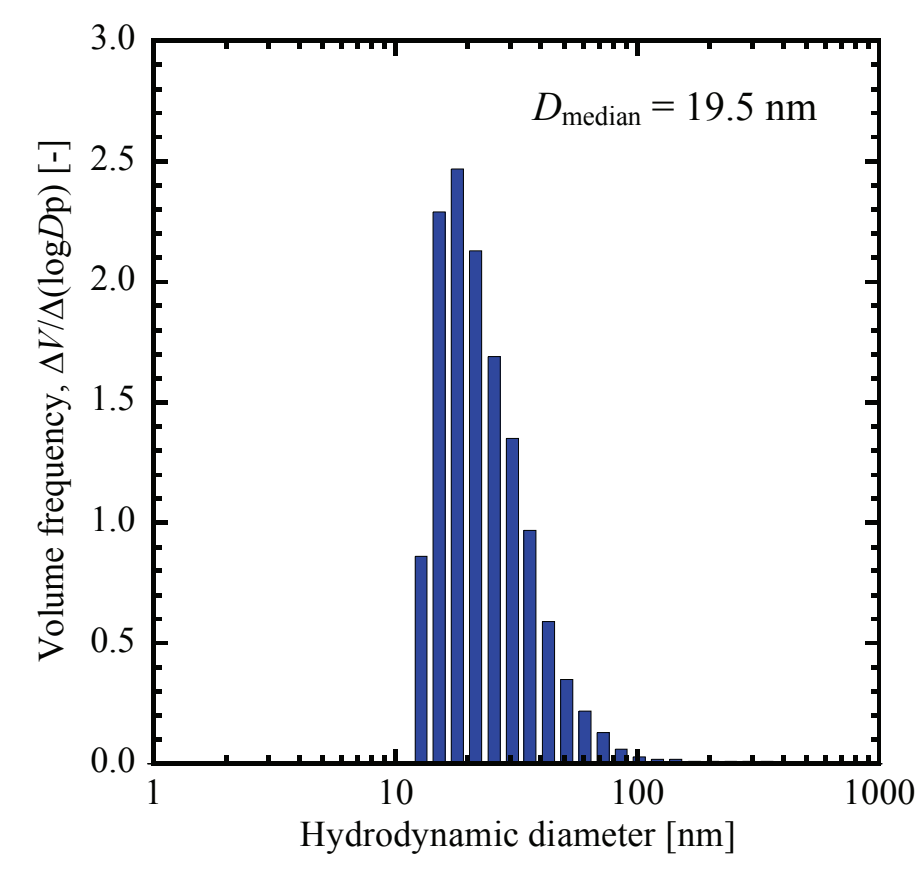

Figure S1. Particle size distribution of the colloidal calcium carbonate particles dispersed in the supernatant. 


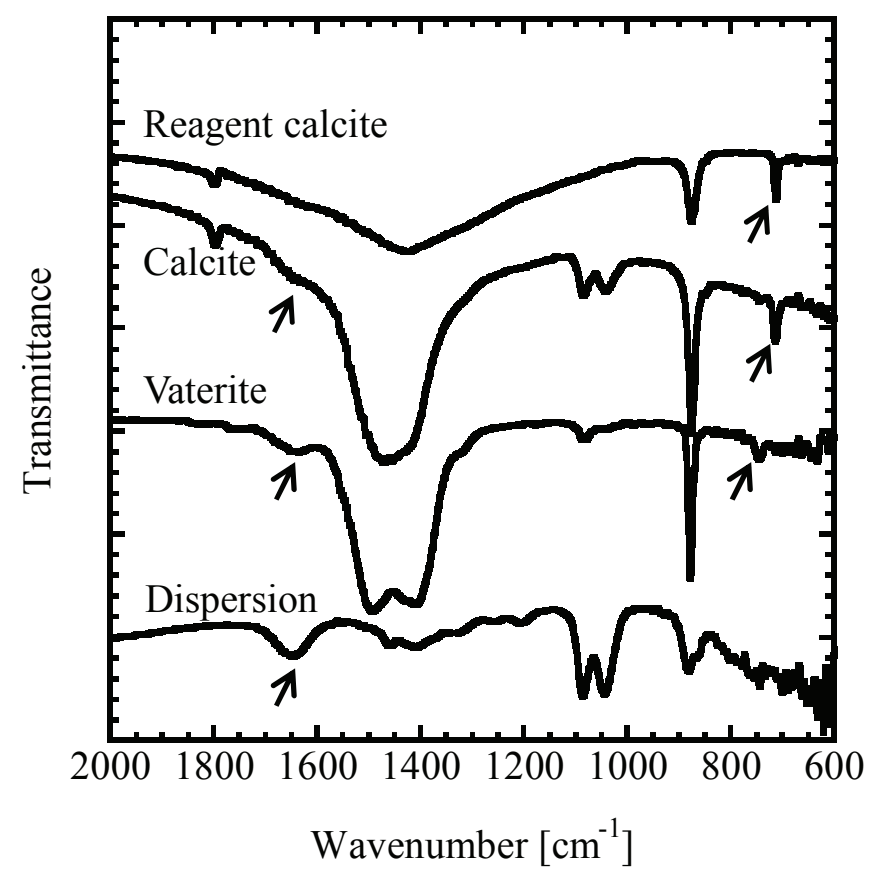

Figure S2. FTIR spectra of the colloidal dispersion, mesoporous vaterite ( 24 hours agitation at $300 \mathrm{rpm}$ ), mesoporous calcite (without agitation), and reagent calcite powder. 


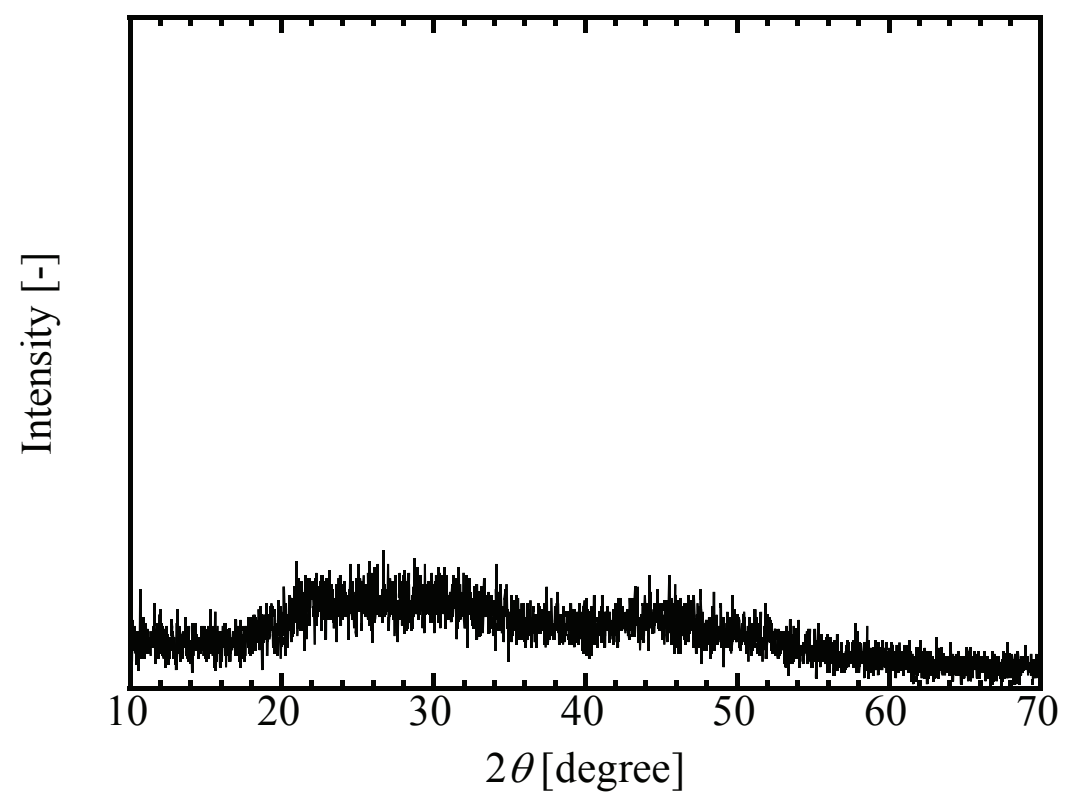

Figure S3. XRD pattern of the solid sample separated from the dispersion. 




Figure S4. XRD pattern of the prepared particles after shaking the colloidal dispersion for 24 hours using a reciprocal shaker at $100 \mathrm{rpm}$ and subsequent aging treatment. 
Graphical and Textual abstract

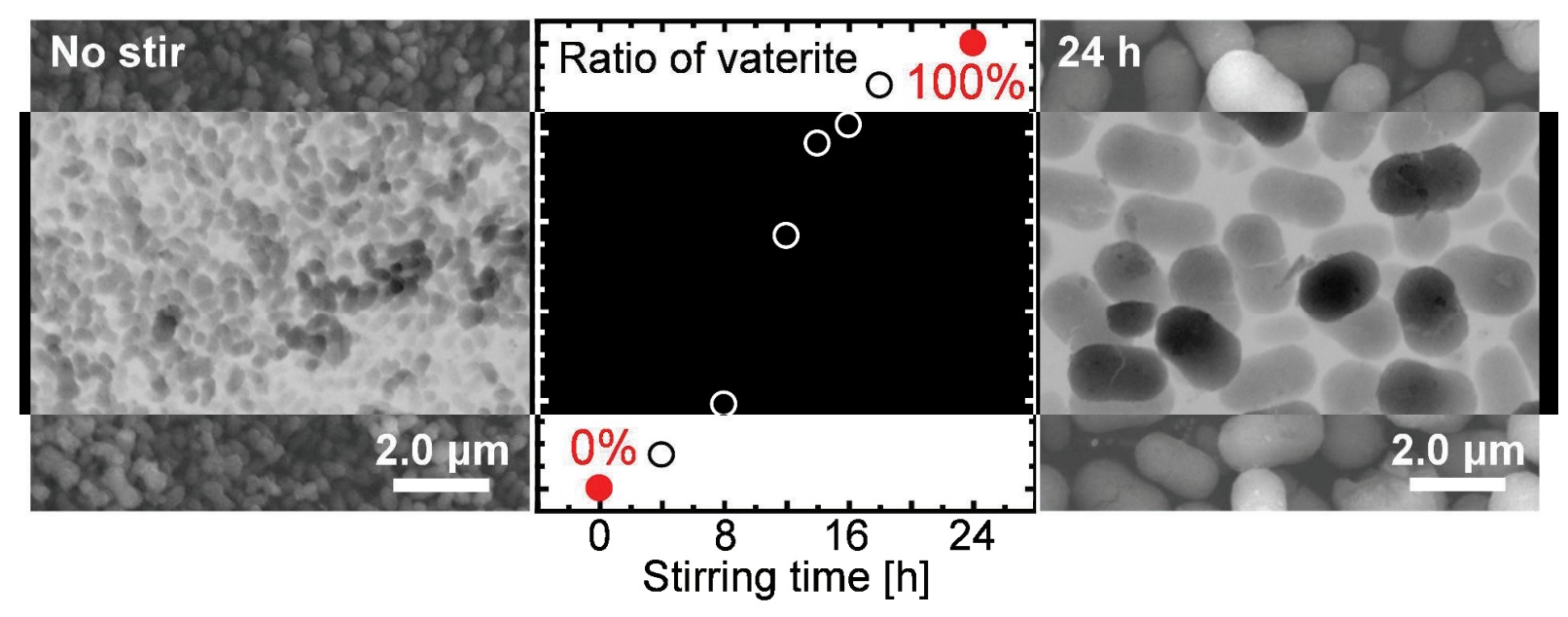

Mechanically stirring the precursor colloidal dispersion can control the polymorphs of mesoporous calcium carbonate. 